\title{
Brain Tumor Boundary Detection in MR Image with Generalized Fuzzy Operator
}

\author{
C.C. Leung ${ }^{1}$, W.F. Chen ${ }^{2}$, P.C.K. Kwok ${ }^{3}$, and F.H.Y. Chan ${ }^{1}$ \\ 'Department of Electrical \& Electronic Engineering, The University of Hong Kong, PRC \\ ${ }^{2}$ Department of Biomedical Engineering, First Military Medical University, PRC \\ ${ }^{3}$ School of Science \& Technology, The Open University of Hong Kong, PRC
}

\begin{abstract}
Boundary detection in MR image with brain tumor is an important image processing technique applied in Radiology for 3D reconstruction. The nonhomogeneities density tissue of the brain with tumor can result in achieving the inaccurate location in any boundary detection algorithms. Recently, some studies using the contour deformable model with regional base technique, the performance is insufficient to obtain the fine edge in the tumor, and the considerable error in accuracy is existed. Moreover, even in some of the normal tissue region, edge created by this method has also been encompassed. In this paper, we propose a new approach to detect the boundary of brain tumor based on the generalized fuzzy operator (GFO). One typical example is used for evaluating this method with the contour deformable model.
\end{abstract}

Keywords - Brain tumor, non-homogeneities density, generalized fuzzy operator.

\section{INTRODUCTION}

In 3D reconstruction of MR image with brain tumor, boundary detection in each 2D image slice of magnetic resonance (MR) can be located by the features in the edge of the brain tumor. Hence, accuracy and sensitivity of the edge in the boundary detection are the two factors to be considered in each of edge detection methods. The accuracy of the edge is in term of the area of the tumor. If the edge is out of the tumor, then, the difference value between the area obtained from edge detection and the real area of tumor is signed on "+". In opposite way, the difference value is signed on "-". For the sensitivity of the edge, the fine edge denotes one pixel in the width of the edge. If more than one, the worst sensitivity of the edge is obtained. Now, we are based on these two factors to measure which methods can be satisfied in edge detection for the nonhomogeneities density tissue of brain tumor.

Tissue inhomogeneity [1] as an additional noise surrounds the tumor is the problem for searching the boundary of tumor. Previous studies [2-4] proposed the contour deformable model (snake) and adaptive thresholding to solve this problem in MR image. However, these kinds of the methods cannot detect the boundary reliably. In fact, the sensitivity of the boundary found by the snake [5] is better than the conventional edge detection methods, such as the Sobel and Laplacian. However, the external energy of snake function is only positive in homogeneous region, and zero at the edges [6]. It is invalid in the nonhomogeneous region while the term of external energy attracts the contour toward the object in the image. Now, we propose a simple but efficient edge detection method that is based on Generalized Fuzzy Operator (GFO) [7]. The special properties of this GFO can search the boundary in high accuracy and obtain the fine edge based on its generalized fuzzy set. Evaluation between Contour Deformable Model (CDM) and our method with MR image are also presented.

\section{The Contour Deformable Model}

Kass et al [5] modeled the contour as an energy minimizing spline guided by internal and external forces. The internal energy part is used to control the smoothness of the contour with weights. The external energy $E_{e x t}$ is from the image edge information, so that

$$
E_{\text {ext }}=-\gamma(s)|\nabla X|^{2}
$$

where $\nabla X$ is the image gradients and $\gamma(s)$ is the weight. Various edge detection operators can be used to construct the external force. The second-order derivatives of the image gradients can also be used to construct $E_{\text {ext }}$

$$
E_{e x t}=-\gamma(s)\left(G_{\sigma}^{*} \nabla^{2} X\right)^{2}
$$

where $G_{\sigma}$ is a Gaussian function of the standard deviation $\sigma$. Minimizing the snake energy will smooth out the contour and move it to nearby edges. A region-based model is formed before the active contour 
is applied. According to the Kolmogorov-Smirnov test method [8], the initial region plan covers a region somewhat different from the desired object when Kolmogorov-Smirnov distance is $F_{o} \neq F_{B}$. A normal shrinking algorithm [9] is used to deform the region to until $F_{D}=F_{B}$. If $F_{o}=F_{B}$, the region becomes the boundary of the object. The advantage of this algorithm is that it will be free from influence from the other regions.

\section{Definitions of GFO}

Definition 1. Denoted Generalized Fuzzy Set (GFS) S in the region $\mathrm{R}$,

$$
S=\int \frac{\mu_{\mathrm{s}}(x)}{x}, x \in R
$$

where $\mu_{S}(x) \in[-1,1]$ is called the Generalized membership function (GMF) of $\mathrm{S}$ on $\mathrm{R}$; for $\mu_{S}(x) \in[-1,0)$, the GMF of $\mathrm{x}$ in $\mathrm{S}$ is not a subordinate on $\mathrm{R}$; for $\mu_{S}(x) \in[0,1]$, the GFM of $\mathrm{x}$ in $\mathrm{S}$ is subordinate on $\mathrm{R}$; and $\mu_{S}(x)=0$, the fuzzy bound point function (FBF) in $\mathrm{S}$ is on $\mathrm{R}$.

According to definition shown in Chen et al, we can write,

$$
\begin{aligned}
& \mu_{\mathrm{T}}(\mathrm{x})=\mathrm{GFO}\left[\mu_{\mathrm{s}}(\mathrm{x})\right]= \\
& \left\{\begin{array}{l}
\sqrt[\beta]{1-\left[1+\mu_{S}(x)\right]^{\beta}},-1 \leq \mu_{S}<0 \\
{\left[\mu_{S}(x)\right]^{\beta}, 0 \leq \mu_{S}<r} \\
\sqrt[\beta]{1-\alpha\left[1-\mu_{S}(x)\right]^{\beta}}, r \leq \mu_{S} \leq 1
\end{array}\right.
\end{aligned}
$$

In this formula, we can deduce the properties shown as below:

Property 1 When $\beta \rightarrow \infty$,

$$
\mu_{7}(x)=\left\{\begin{array}{l}
1,-1 \leq \mu_{S}(x)<0 \\
0,0 \leq \mu_{S}(x)<r \\
1, r \leq \mu_{S}(x) \leq 1
\end{array}\right.
$$

Property 2 When $\beta>1$,
$\left\{\begin{array}{l}\mu_{T}(x)>\mu_{S}(x), \text { if }-1 \leq \mu_{S}(x)<0, r<\mu_{S}(x) \leq 1, \\ \mu_{T}(x)<\mu_{S}(x), \text { if } 0<\mu_{S}(x) \leq r\end{array}\right.$

Now, the generalized fuzzy set $S$ becomes to a normal fuzzy set $T$.

\section{Edge detection using a modified Generalized Fuzzy Operator}

From section 3, we use the GFO to map all the pixels in the original image into the GFS. For an image $X(i, j)$, where $i=1,2, \ldots, N$ and $j=1,2, \ldots, M$.

We use a Sine function to change $X(i, j)$ into a fuzzy set $P(i, j)$ :

$$
P(i, j)=\sin \left\{\frac{\pi}{2}\left(1-\frac{X(i, j)-X_{\min }}{D}\right)\right\}
$$

where $\frac{X_{\max }-X_{\min }}{2} \leq D$

then, $P \in[-1,1]$ is mapped to the new fuzzy set $P$, shown in the following equation.

$$
P^{\prime}(i, j)=\left\{\begin{array}{l}
\sqrt[\beta]{\left(1-(1+P(i, j))^{\beta}\right.},-1 \leq P<\gamma \\
1, P=\gamma \\
\sqrt[\beta]{\left(1-\alpha(1-P(i, j))^{\beta}\right.}, \gamma<P \leq 1
\end{array}\right.
$$

We set $\beta=2$, and the newer image $X^{\prime}(i, j)$ is

$$
X^{\prime}(i, j)=X_{\min }+D\left\{1-\left[\frac{\sin ^{-1}\left(P^{\prime}(i, j)\right)}{\frac{\pi}{2}}\right]\right\}
$$

The fuzzy set $P^{\prime}(i, j)$ includes three conditions. In the range of $[-1, \gamma)$, we assigned it as the gray scale 
lower than the desired gray-level. The second term, $P$ $=\gamma$, was the desired gray-level (the edge of object). The last term, the range is $(\gamma, 1]$, was the gray-level higher than the desired gray-level. The characteristic of the modified GFO is a "V" shape curve located in [-1 1] of $P(i, j)$. This function response likes as a band-pass function that the useful gray-level should have mapped to a gray-level mostly difference with the non-useful. The parameter " $\gamma$ " can be adjusted to shift the " $V$ " type function along the $P(i, j)$ axis, depending on the actual gray levels of the boundary of interest. " $\alpha$ " can affect the right arm of the "V" type function. This function can contribute the boundary searching so that the edge of the tumor can be located. The second character of the modifed GFO is related to the mapping. The original image $X(i, j)$ is mapped into the new image $X^{\prime}(i, j)$. In the new mapping, it is compressed to a certain gray-level range according to the three fuzzy conditions and the parameter $D$ introduced in the equation (4-1). The result of the new mapping will have an enhancement function so that the object locates in the higher gray-level will be changed to a lower gray-level. However, the background gray-level will be mapped separated from that object. Enhancement can be derived.

\section{Results and Discussion}

We implement our method with contour deformable model (CDM) in a Pentium II PC. The algorithms are programmed in the MATLAB. One MR image with the brain tumor in the frontal has been used to evaluate our method and the CDM. Figure 1 shows the original MR image with brain tumor. The tumor is spread out in the frontal of the brain and is tissue inhomogeneity.

For the CDM with the regional base [3], it is assumed that brain tumor is a solid in which can be regarded as a region that is homogeneous in some properties. The property with some disparity between the object and its surroundings should be chosen to identify the object. The boundary of the object is a sub-region of the object that borders other objects. It is based on an initial estimation of the object, the goal of region deforming is to find a maximum area region of which the boundary has the same properties as the region. However, the edge generated by the CDM in MR image cannot match to the boundary of the brain tumor perfectly since the tumor is tissue non-homogeneities density. The result of using CDM to generate the edge in the evaluation data is shown in Figure 2. It shows that some of the normal soft tissue in the brain has been involved within the region created by the CDM. To measure the accuracy of the edge, the difference in the total number of pixels between original edge and the edge created by CDM and mGFO are shown in Table 1. It is " +694 " pixels over 1689 pixels that come from the original edge, $41.09 \%$ error in matching for using CDM. Other than the region out of the tumor, a small region of normal tissue has been encompassed in the tumor in which need not be counted in the total pixel number of tumor region. It has totally "-60" pixels been obsolete.

For the modified GFO, the fuzzy set can separate the edge, normal tissue and tumor respectively. Equation (4-3) can re-map pixels of the normal tissue and tumor into the higher gray scale level. By then, only edge is set into zero. Hence, edge can be extracted when all the lowest gray scale pixels transform to " 0 ", and the other pixels transform to " 1 ". In the measurement of using the modified GFO, it is totally " +34 " pixels over 1689 pixels that come from original edge. There is $2 \%$ error in matching for using our method. For the small region of normal tissue located within the tumor, "- 4 " pixels have been obsolete within the tumor. Consequently, based on the frontal brain tumor, the boundary detection using our method is better than the method of CDM.

\section{Conclusion}

The modified Generalized Fuzzy Operator has been presented. It is a simple but effective method using in boundary detection. As shown in the example, it is very accurately in searching the boundary of the brain tumor, and is a useful method in pre-processing for $3 \mathrm{D}$ reconstruction.

\section{Reference}

[1] W.J.H. Veldkamp, and N. Karssemeijer, "Normalization of Local Contrast in Mammograms," IEEE Trans. on Medical Imaging, Vol.19, No.7, pp.731-738, July 2000.

[2] Y. Zhu, and H. Yan, "Computerized tumor boundary detection using a Hopfield neural network," IEEE Trans. on Medical Imaging, Vol.16, No.1, pp.5567, 1997.

[3] F.H.Y. Chan, F.K. Lam, P.W.F. Poon, H. Zhu, and K.H. Chan, "Object boundary location by region and contour deformation," IEE Proceedings Vision, Image 
and Signal Processing, Vol.143, No.6, pp.353-360, Dec., 1996.

[4] D.J. Kang, "Stable snake algorithm for convex tracking of MRI sequences," Electron. Lett., Vol.35, pp.1070-1071, 1999.

[5] M. Kass, A. Witkin, and D. Terzopoulos, "Snake: active contour models," in Proc. of the First International Conf. on Computer Vision, pp.259-268, 1987.

[6] T.F. Chan, and L.A. Vese, "Active Contours without Edges," IEEE Trans. on Image Processing, Vol.10, No.2, pp.266-277, Feb., 2001.

[7] W.F. Chen, X.Q. Lu, J.J. Chen, and G.X. Wu, "A new algorithm of edge detection for color image: Generalized Fuzzy Operator," Science in China (Series A), Vol. 38, No. 10, pp.1272-1280, 1995.

[8] I. Pitas, Digital image processing algorithms, Prentice Hall, USA, 1993.

[9] J. Gutierrez, I. Epifanio, E. De Ves, and F. Ferri, "An active contour model for the automatic detection of the Fovea in Fluorescein Angiographies," in Proc. Internation Conf. on Pattern Recog., Vol.4, pp.312315, Sept., 2000.

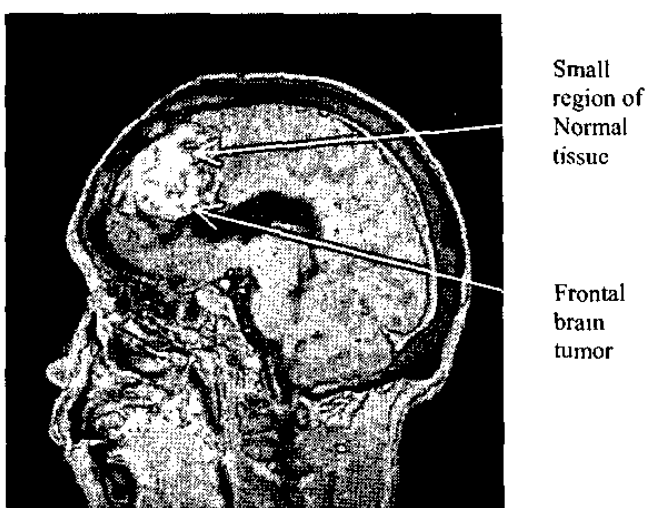

Figure 1. The original MR image with the frontal brain tumor.

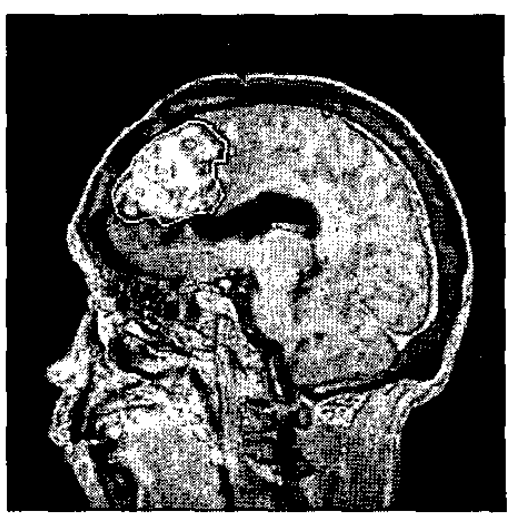

Figure 2, The edge is generated by the CDM. Some tissue out of the tumor is involved.

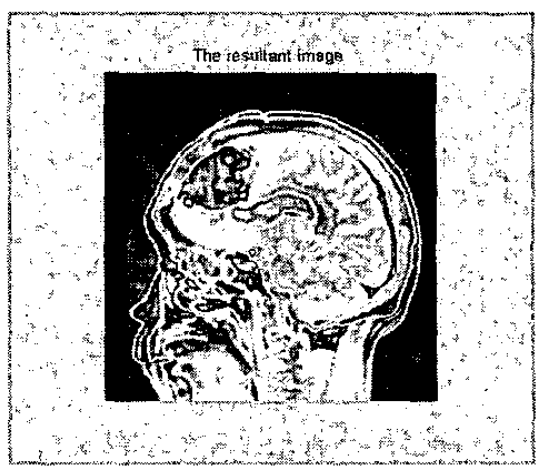

Figure 3. The edge is generated by the modified GFO Almost most of the tumor tissue is included in the edge. 